\title{
Joint Controller-Communication Topology Design for Distributed Wide-Area Damping Control of Power Systems
}

\author{
Junqi Liu* Azwirman Gusrialdi ${ }^{* *}$ Sandra Hirche ${ }^{* *}$ \\ Antonello Monti * \\ * E.ON Energy Research Center, RWTH Aachen University, \\ Mathieustr. 6, 52074 Aachen, Germany, \\ ([jliu,amonti]@eonerc.rwth-aachen.de) \\ ** Institute of Automatic Control Engineering, Technische Universität \\ München, D-80290 Munich, Germany, (iman@lsr.ei.tum.de, \\ hirche@tum.de)
}

\begin{abstract}
The recent development and deployment of the synchronized phasor measurement units (PMU) is allowing the wide-area monitoring and control of large-scale power systems. Furthermore, the integration of more communication technologies into the power system is giving an additional degree of freedom to the control design that may improve the performance of the overall system. Small-signal stability is an important requirement for power systems with the increasing number of distributed generation units. The oscillation modes of the power system have to be well damped in order to avoid contingencies such as blackouts. Wide-area controllers based on the real-time PMU measurements operating in centralized, distributed and decentralized manner have been widely proposed to damp the low-frequency oscillation of the large-scale interconnected power system. It has been shown that the damping performance can be improved by using the synchronized PMU data transmitted in real-time via communication network. However, only a few of the proposed methods take the structural constraint of the measurement data transmission into account. In this paper, we propose a method to design a distributed wide-area damping controller together with the communication topology in order to improve the damping performance of the power system. As a design strategy, first a decentralized controller that stabilizes the overall system is designed. Then, the damping performance is improved by designing the distributed control law, i.e. allowing the local controllers to exchange information. The problem is formulated as a mixed-integer optimization. Finally the proposed approach is evaluated in a five machine power system via a numerical simulation.
\end{abstract}

Keywords: Power system control, wide-area power system control, distributed control, optimization

\section{INTRODUCTION}

With the deregulation and integration of large amount of distributed generation units, power systems are increasingly driven to operate closer to their operating and stability limits. Under critical operation conditions in which the possibility of low-frequency oscillations rises, the smallsignal stability of the system has to be guaranteed for secure operation. These low-frequency oscillation modes have to be well damped by applying advanced control strategies in order to avoid fatal contingencies such as blackouts.

The contemporary solution is the combined Automatic Voltage Regulator (AVR) - Power System Stabilizers (PSS) approach with the support of wide-area signals. Such controllers are usually designed based on pole placement and phase compensation technique in terms of increasing the damping ratio or the decay rate of the system in response to small disturbances. The development and deployment of Wide-Area Measurement System (WAMS) based on the synchronized phasor measurement units (PMU) technology [Phadke and Thorp (2008), De La Ree et al. (2010)] enables the remote measurement and transmission of the synchronized system dynamic data such as voltage, angle, frequency from and to different locations. Wide-Area Control System (WACS) using the PMU measurements for the oscillation damping have been widely investigated. It is shown that with the remote measured signals, the wide-area controller improves the damping performance and the stability of the power system [Rostamkolai et al. (1988), Aboul-Ela et al. (1996), Wu et al. (2004), Snyder et al. (2000)].

Different control structures have been proposed for the WACS design. In [ Snyder et al. (2000), Ni et al. (2002), Wu et al. (2004)] a centralized controllers have been designed as a supervisory instance to collect remote signals from each local measurement units and send the processed control signals back to local AVR of the generators. Two-level hierarchical control have also been widely investigated, see e.g. [Kamwa et al. (2001), Zhang and 
Bose (2008), Dotta et al. (2009), Hashmani and Erlich (2008)]. In such a structure, a decentralized control serves as the first level, comprehending the local controller at generators or FACTS (Flexible AC Transmission System) equipments, and provides the stabilizing action for local oscillation modes for which global information may not be necessary. It also ensures the system stability during events of loss of communication links or of failure that makes the central instance unavailable. A centralized controller, as the second control level, collects and processes the widearea measurement data. Its output is transmitted to the local controllers of the generators in order to improve the global system performance.

In any case, reliable and efficient communication networks are required for enabling the WAMS and the WACS. In the aforementioned works, the topology of the communication network is fixed for the controller design. The integration of communication technologies into the power system gives though an additional degree of freedom in designing the distributed controller for the large-scale systems and can be used to improve the performance of the overall systems under a given communication network's constraint.

In this paper, we propose a design procedure for the wide-area power system damping control from a new perspective. Based on the theoretical analysis in [Gusrialdi and Hirche (2010)], we design a distributed controller in terms of a joint controller gain and communication topology design approach by exploring the additional degrees of freedom offered by the communication network. Another design objective is to increase the robustness of the overall system in the presence of the permanent communication links failures. In order to achieve the goals, first we design a decentralized controller that ensures the stability of the overall power system. Then, we extend the decentralized controller to a distributed one by enabling communication, in this case the exchange of the state information, between the local controllers. The problem is formulated as a mixed integer optimization problem.

The remainder of the paper is organized as follows. The model of the power system used in the paper is described in Section 2. The design procedure for the joint controller gain and communication topology is reviewed in Section 3 . The proposed distributed damping control is discussed in Section 4. Finally, the proposed method is evaluated via a numerical simulation in a five machine power system in Section 5 .

\section{POWER SYSTEM MODELING}

Generally, a power system is an interconnected system and its model consists of differential and algebraic equations describing the generator dynamics, controllers, networks and loads. In terms of the small-signal stability and damping control for low-frequency oscillations, a linearized model including the generator dynamics and an equivalent transfer network model is considered. The equivalent transfer network is a reduced network model in which all load nodes are eliminated and all generator nodes are directly connected with each other, see e.g. [Machowski et al. (2008)]. In this paper we consider a five-machine power system [Wu and Malik (2006)] as a case study as shown in Fig. 1. The system considered in this paper

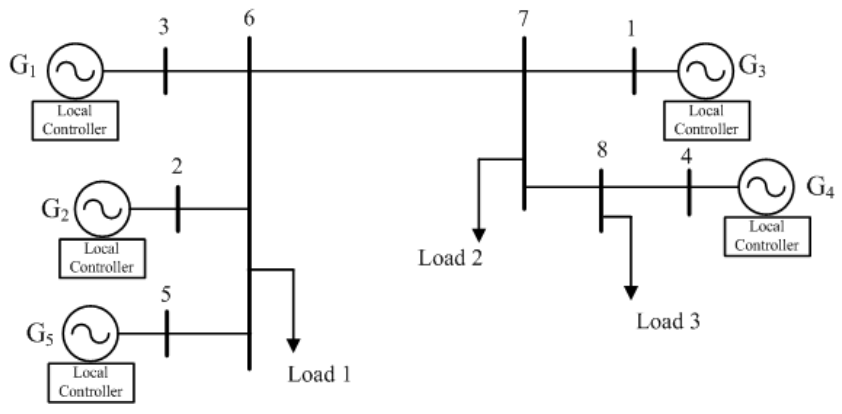

Fig. 1. A five machine power system for case study in this paper

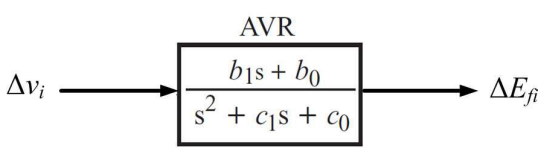

Fig. 2. Automatic voltage regulator model

consists of the generator model, excitation system with voltage control and the network model.

\subsection{Generator and exciter system model}

A linearized third order generator model with an excitation system as introduced in [Machowski et al. (2008)] is used here as the model of $i$-th generator in the system. The nomenclature of all the variables and parameters can be found in Appendix A.

- Generator dynamic equations:

$$
\begin{aligned}
\Delta \dot{\delta}_{i} & =\Delta \omega_{i} \\
M_{i} \Delta \dot{\omega}_{i} & =-D_{i} \Delta \omega_{i}-\Delta P_{e i} \\
T_{d o i}^{\prime} \Delta \dot{E}_{q i}^{\prime} & =-\Delta E_{q i}^{\prime}+\left(X_{d i}-X_{d i}^{\prime}\right) \Delta I_{d i}+\Delta E_{f i}
\end{aligned}
$$

A second-order transfer function is used to represent the Automatic Voltage Regulator (AVR) for the generator exciter as shown in Fig. 2. An AVR controls the excitation current, and consequently the generators terminal voltage.

- Excitation system and automatic voltage control

$$
\begin{aligned}
\dot{z}_{2 i} & =-c_{1 i} z_{2 i}-c_{0 i} z_{1 i}+\Delta v_{i}, \\
\dot{z}_{1 i} & =z_{2 i}, \\
\Delta E_{f i} & =b_{1 i} z_{2 i}+b_{0 i} z_{1 i},
\end{aligned}
$$

where $z_{1 i}$ and $z_{2 i}$ are the internal states of the AVR.

The linearized state dynamic $\mathbf{x}_{i}$ of the $i$-th synchronous generator is then given by

$$
\mathbf{x}_{i}=\left[\begin{array}{lllll}
\Delta \delta_{i} & \Delta \omega_{i} & \Delta E_{q i}^{\prime} & z_{2 i} & z_{1 i}
\end{array}\right]^{T}
$$

and the control input signal is denoted by $\Delta v_{i}$.

\subsection{Network Model}

In general, the network model of a power system is represented by the algebraic nodal equations describing the relation between the current injections and the voltages of all generation and load nodes via the admittance matrix. As discussed in [Machowski et al. (2008)], eliminating the load nodes leads to an equivalent transfer network in which the generator nodes are directly connected with each other. 
After the elimination of all the load nodes, including the generation terminal nodes, the d-axis currents $I_{d i}$ and the electrical power $P_{e i}$ of the $i$-th generator can be expressed in terms of its variables, in this case the rotor angle $\delta_{i}$ and the transient voltage $E_{q i}^{\prime}$ according to

$$
\begin{aligned}
& I_{d i}=\sum_{j=1}^{N} E_{q i}^{\prime}\left[B_{i j} \cos \left(\delta_{i}-\delta_{j}\right)-G_{i j} \sin \left(\delta_{i}-\delta_{j}\right)\right] \\
& P_{e i}=E_{q i}^{\prime} \sum_{j=1}^{N}\left[B_{i j} \sin \left(\delta_{i}-\delta_{j}\right)+G_{i j} \cos \left(\delta_{i}-\delta_{j}\right)\right] E_{q j}^{\prime}
\end{aligned}
$$

where $N$ is the total number of generators in the system and $i, j \in\{1 \cdots N\}, B_{i j}$ and $G_{i j}$ are the imaginary and the real part of a network admittance $Y_{i j}$.

Linearizing the equations (8) and (9), the power increment $\Delta P_{e i}$ in (2) and the current increment $\Delta I_{d i}$ in (3) are given by

$$
\begin{gathered}
\Delta P_{e i}=\left[\begin{array}{ll}
\frac{\partial P_{e i}}{\partial \boldsymbol{\delta}} & \frac{\partial P_{e i}}{\partial \mathbf{E}_{q}^{\prime}}
\end{array}\right]\left[\begin{array}{c}
\Delta \boldsymbol{\delta} \\
\Delta \mathbf{E}_{q}^{\prime}
\end{array}\right], \\
\Delta I_{d i}=\left[\begin{array}{ll}
\frac{\partial I_{d i}}{\partial \boldsymbol{\delta}} & \frac{\partial I_{d i}}{\partial \mathbf{E}_{q}^{\prime}}
\end{array}\right]\left[\begin{array}{c}
\Delta \boldsymbol{\delta} \\
\Delta \mathbf{E}_{q}^{\prime}
\end{array}\right]
\end{gathered}
$$

with $\Delta \boldsymbol{\delta}$ consists of the rotor angle deviations and $\Delta \mathbf{E}_{q}^{\prime}$ consists of the transient voltage deviations of all generators in the power systems.

Combining the equation (1)-(6) and (10)-(11), the smallsignal dynamics of a generator connected to the power system can be written as

$$
\dot{\mathbf{x}}_{i}=\mathbf{A}_{i} \mathbf{x}_{i}+\mathbf{B}_{i} u_{i}+\sum_{j \in N_{i}} \mathbf{A}_{i j} \mathbf{x}_{j}
$$

where $u_{i}=\Delta v_{i}$ and $N_{i}$ represents the set of the generators that are physically connected with the $i$-th generator. The matrices $\mathbf{A}_{i} \in \mathbb{R}^{5 \times 5}, \mathbf{B}_{i} \in \mathbb{R}^{5 \times 1}, \mathbf{A}_{i j} \in \mathbb{R}^{5 \times 5}$ are given in Appendix B.

Therefore, the linearized power system can be expressed by an LTI interconnected systems given by

$$
\dot{\mathbf{x}}=\mathbf{A} \mathbf{x}+\mathbf{B u},
$$

where $\mathbf{x} \in \mathbb{R}^{5 N \times 1}$ and $\mathbf{u} \in \mathbb{R}^{N \times 1}$ are the states and input signals of all $N$ generators in the power system respectively and the matrices $\mathbf{A} \in \mathbb{R}^{5 N \times 5 N}, \mathbf{B} \in \mathbb{R}^{5 N \times N}$ of appropriate dimensions are given by

$$
\mathbf{A}=\left[\begin{array}{cccc}
\mathbf{A}_{1} & \mathbf{A}_{12} & \cdots & \mathbf{A}_{1 N} \\
\mathbf{A}_{21} & \mathbf{A}_{2} & \cdots & \mathbf{A}_{2 N} \\
\vdots & \vdots & \ddots & \vdots \\
\mathbf{A}_{N 1} & \mathbf{A}_{N 2} & \cdots & \mathbf{A}_{N}
\end{array}\right], \quad \mathbf{B}=\left[\begin{array}{cccc}
\mathbf{B}_{1} & \mathbf{0} & \cdots & \mathbf{0} \\
\mathbf{0} & \mathbf{B}_{2} & \ddots & \vdots \\
\vdots & \ddots & \ddots & \mathbf{0} \\
\mathbf{0} & \cdots & \mathbf{0} & \mathbf{B}_{N}
\end{array}\right]
$$

\section{JOINT CONTROLLER AND COMMUNICATION TOPOLOGY DESIGN}

In this section we review the procedure for designing the distributed controller together with the communication topology proposed in [Gusrialdi and Hirche (2010)] for the interconnected system given by (12) in order to improve the performance of the system. As a performance metric, the decay rate of the overall systems is considered. Here we consider a state feedback controller for which the control law can be written as follows.

$$
u_{i}=\mathbf{K}_{i} \mathbf{x}_{i}+\sum_{j \in L_{i}} \mathbf{K}_{i j} \mathbf{x}_{j}
$$

which is known as distributed control law since the controller for each subsystem does not only depend on its own states but also on the states of the other subsystems. Here $L_{i}$ represents a set of subsystems to which controller $i$ could communicate, i.e., exchange information. If $\mathbf{K}_{i j}=0, \forall i$ and $\forall j \in L_{i}$, then the control law is called a decentralized control law.

\subsection{Decentralized control law design}

As a design strategy, first we design the decentralized control law using the standard methods that stabilize the interconnected systems (12), i.e., we consider the decentralized controller synthesis for the interconnected systems (12) with the control input given by

$$
\tilde{u}_{i}=\mathbf{K}_{i} \mathbf{x}_{i} \text {. }
$$

Let $\mathbf{A}_{i}+\mathbf{B}_{i} \mathbf{K}_{i}=\overline{\mathbf{A}}_{i}$. The closed loop expression of the interconnected systems (12) with the decentralized control law can be written as

$$
\dot{\mathbf{x}}=\mathbf{A}_{\mathrm{dec}} \mathbf{x}, \mathbf{x}\left(t_{0}\right)=\mathbf{x}_{0},
$$

where $\mathbf{x}=\left[\mathbf{x}_{1}, \mathbf{x}_{2}, \cdots, \mathbf{x}_{N}\right]^{T}$ and

$$
\mathbf{A}_{\mathrm{dec}}=\left[\begin{array}{cccc}
\overline{\mathbf{A}}_{1} & \mathbf{A}_{12} & \cdots & \mathbf{A}_{1 N} \\
\mathbf{A}_{21} & \overline{\mathbf{A}}_{2} & \cdots & \mathbf{A}_{2 N} \\
\vdots & \vdots & \ddots & \vdots \\
\mathbf{A}_{N 1} & \mathbf{A}_{N 2} & \cdots & \overline{\mathbf{A}}_{N}
\end{array}\right] \in \mathbb{R}^{5 N \times 5 N}
$$

It is well known that the solution of (16) is given by $\mathbf{x}(t)=e^{\mathbf{A}_{\mathrm{dec}}\left(t-t_{0}\right)} \mathbf{x}_{0}$ and the state norm satisfies

$$
\|\mathbf{x}(t)\| \leq e^{\left\|\mathbf{A}_{\mathrm{dec}}\right\|\left(t-t_{0}\right)}\left\|\mathbf{x}_{0}\right\|, \forall t \geq t_{0},
$$

and

$$
\|\mathbf{x}(t)\| \leq e^{\operatorname{Re}\left\{\lambda_{\max }\left(t-t_{0}\right)\right\}}\left\|\mathbf{x}_{0}\right\|, \forall t \geq t_{0},
$$

where $\operatorname{Re}\left\{\lambda_{\max }\right\}$ represents the real part of the largest eigenvalues of $\mathbf{A}_{\mathrm{dec}}$.

\subsection{Distributed control law design}

Let us assume that the decentralized control law stabilizing the interconnected system (12), i.e., the first term of (14), has been designed. Next we improve the performance of the systems for a certain performance metric by designing the distributed controller, that is the feedback gain and the communication topology.

The objective is to improve the performance of the overall systems, i.e., increase the convergence rate by designing the second term of (14) given by the following controller

$$
\bar{u}_{i}=\sum_{j \in L_{i}} d_{i j} \mathbf{K}_{i j} \mathbf{x}_{j}
$$

where $d_{i j} \in\{0,1\}$ is a binary number that shows the possibility to perform the state information exchange between controller $i$ and $j$, i.e., $d_{i j}=1$ means that a communication link is added between the local controllers $i$ and $j$ and vice versa. The new closed loop expression of (12) with the addition of controller (19) is given by

$$
\dot{\mathbf{x}}=\overline{\mathbf{A}} \mathbf{x}, \mathbf{x}\left(t_{0}\right)=\mathbf{x}_{0},
$$




$$
\begin{aligned}
\overline{\mathbf{A}} & =\left[\begin{array}{cccc}
\overline{\mathbf{A}}_{1} & \mathbf{A}_{12} & \cdots & \mathbf{A}_{1 N} \\
\mathbf{A}_{21} & \overline{\mathbf{A}}_{2} & \cdots & \mathbf{A}_{2 N} \\
\vdots & \vdots & \ddots & \vdots \\
\mathbf{A}_{N 1} & \mathbf{A}_{N 2} & \cdots & \overline{\mathbf{A}}_{N}
\end{array}\right]+\left[\begin{array}{cccc}
\mathbf{0} & \overline{\mathbf{A}}_{12} & \cdots & \overline{\mathbf{A}}_{1 N} \\
\overline{\mathbf{A}}_{21} & \mathbf{0} & \cdots & \overline{\mathbf{A}}_{2 N} \\
\vdots & \vdots & \ddots & \vdots \\
\overline{\mathbf{A}}_{N 1} & \overline{\mathbf{A}}_{N 2} & \cdots & \mathbf{0}
\end{array}\right] \\
\overline{\mathbf{A}} & =\mathbf{A}_{\text {dec }}+\mathbf{A}_{\text {dist }}
\end{aligned}
$$

The matrix $\mathbf{A}_{\text {dec }}$ is the closed-loop system with the decentralized control in (16), thus, the matrix $\mathbf{A}_{\mathrm{dec}}$ is stable. The term $\overline{\mathbf{A}}_{i j}$ is defined as $\overline{\mathbf{A}}_{i j}=\mathbf{d}_{i j} \mathbf{B}_{i} \mathbf{K}_{i j}$. Furthermore, we assume that not an arbitrary number of links can be added, i.e., the number is limited by an upper bound induced by the communication constraint

$$
\sum_{1 \leq i \leq j \leq N} \gamma_{i j} d_{i j} \leq c
$$

where $c>0$ is the total cost constraint on the communication network, and $\gamma_{i j}$ represents a cost to establish a link between subsystem $i$ and $j$. This cost is typically related to factors such as the distance between the subsystems.

The problem can then be formulated as finding the gain and topology of the distributed controller such that the convergence rate of the overall interconnected system is optimized under a given communication constraint. The distributed controller is given by the following proposition.

Proposition 1. Considering an interconnected system in (20), if there exists a solution of the optimization problem

$$
\begin{array}{cl}
\underset{\mathbf{K}_{i j}, d_{i j}}{\operatorname{minimize}} & \operatorname{Re}\left\{\lambda_{\max }(\overline{\mathbf{A}})\right\} \\
\text { subject to } & \operatorname{Re}\left\{\lambda_{\max }(\overline{\mathbf{A}})\right\}<\operatorname{Re}\left\{\lambda_{\max }\left(\mathbf{A}_{\mathrm{dec}}\right)\right\}, \\
& \sum_{1 \leq i \leq j \leq N} \gamma_{i j} d_{i j} \leq c \\
& d_{i j} \in\{0,1\},
\end{array}
$$

then the decay rate of the interconnected system with the distributed control law (14) is higher than with the decentralized control law (15) and the whole system remains stable.

The optimization problem (23) is a mixed integer optimization problem since it is solved with respect to both the feedback gain and the communication topology of the distributed controller. The optimization (23) can be solved using well-known techniques such as relaxation and decomposition techniques or cutting planes approaches [Korte and Vygen (2000)].

Remark 2. Since the first term of (14) is designed in advanced and not a part of the decision variables in the optimization problem (23), the performance may not be optimal. However, as shown in [Gusrialdi and Hirche (2010)], the stability of the overall system is guaranteed in the presence of permanent communication links loss. In addition, the optimization problem might be implemented according to a penalty method that will be investigated in future work.

\section{PROPOSED DISTRIBUTED DAMPING CONTROL FOR POWER SYSTEMS}

The conventional method for damping control is based on the modal analysis since the oscillation modes are characterized by the eigenvalues of the linearized power system as formalized in (13).

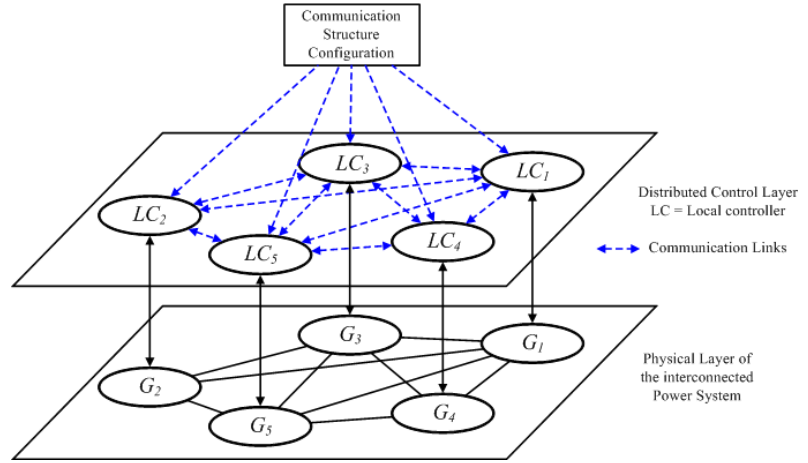

Fig. 3. Proposed distributed control architecture

Let $\lambda_{i}=\alpha_{i}+j \beta_{i}$ be the $i-t h$ eigenvalue of the state matrix $\mathbf{A}$ in (13),

- $\alpha_{i}$ shows the damping or decay rate;

- $\beta_{i}$ shows the frequency of the oscillation;

- the relative damping ratio is given by

$$
\xi_{i}=\frac{-\alpha_{i}}{\sqrt{\alpha_{i}^{2}+\beta_{i}^{2}}} .
$$

The contemporary solution is usually realized based on pole placement and phase lead compensation. The control goal can be formulated as to improve the decay rate of the system oscillations by shifting the real part of the eigenvalue $\alpha_{i}$ towards the left half complex plane that also means to minimize $\alpha_{i}$ which is negative in a system with a stabilizing controller. This method of pole shifting can also be formulated as an optimization problem as in (23). Remark 3. The decay rate of the overall system that is $\operatorname{Re}\left\{\lambda_{\max }\right\}$ is chosen as a performance metric in this paper, since it influences the relative damping ratio of the power system which is the main concern of this paper as will be shown later. Moreover, $\operatorname{Re}\left\{\lambda_{\max }\right\}$ also plays a dominant role in the dynamic responses of the power system [Huang (2006)].

On the other hand, the eigenvalue assignment is proved to be complicated, while the optimal linear quadratic (LQ) control provides a systematic way of designing the feedback controller for the complex systems with high order. Moreover, the resulting LQ controller is guaranteed to stabilize the system with sufficient margins [Aldeen and Crusca (2002)].

Using the proposed design procedure introduced in Section 3, we first design a decentralized LQ controller using the approach discussed in [Liu et al. (2009)] that stabilizes the system and provides a minimum damping performance. Then, the distributed controller together with the optimized communication structure under a given communication network constraint is designed by solving the mixed-integer optimization problem (23) that improves the decay rate, i.e., the damping performance of the overall system.

The control architecture is illustrated in Fig. 3. In contrast to the conventional supervisory centralized controller of the whole system, e.g., [Wu et al. (2004), Snyder et al. (2000)], the central instance in our approach is only responsible for the optimization of the communication topology, i.e. solving the optimization problem (23). After 


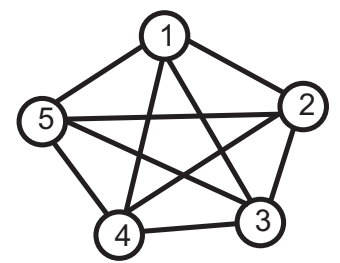

(a)

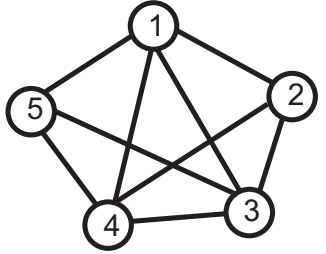

(b)

Fig. 4. (a) Interconnected system used in the simulation; (b) the optimal communication topology

the optimization is performed, the generators with local controllers can operate autonomously while exchanging information with each other. Since it is possible to use any of the variables from the generators, e.g., the generator rotor speeds, angles, voltages or other variables [Hashmani and Erlich (2008)], in this paper it is assumed that the local controller of the generators could measure all of its dynamic states and exchange them with each other via the communication links. Since AVR control loop with wide-area signals could provide relatively good damping performance for the inter-area oscillations of the power system as discussed in [Wu et al. (2004), Snyder et al. (2000)], we consider in this paper that each generator is equipped with a local AVR but no PSS which could further improve the damping performance of the system. However, this does not affect the applicability of the design principle of the proposed approach. The inclusion of PSS will be considered in future work.

\section{EVALUATION AND SIMULATION RESULTS}

The proposed distributed controller is evaluated via a numerical simulation using MATLAB for the power system shown in Fig. 1 by considering the linearized model in (1)-(13). The parameters are chosen as in [Wu and Malik (2006)] and listed in Appendix C.

The system turns out to have a fully connected physical structure, i.e. all the five generators are physically coupled with each other as shown in Fig. 4a. The $\gamma_{i j}$ and $c$ in (22) are equal to 1 and 9 respectively. This means that only 9 communication links are available for the overall system. Solving the optimization problem (23) using the YALMIP toolbox [Lofberg (2004)] gives the optimal topology as shown in Fig. 4b, where all local controllers communicate with each other except between the local controller of generator 2 and 5 .

The eigenvalue analysis of the inter-area oscillations of the test system is compared in Table 1 in terms of the damping ratio and frequencies in the case of with and without the obtained controller. The analysis shows that compared to the open loop, the decentralized controller improved slightly the decay rates and the damping ratios. Note that according to the optimization problem (23), only the maximal real part of the eigenvalues is taken into account to be minimized, which does not necessarily mean that the real parts of all eigenvalues will be minimized, for example the second eigenvalue of the distributed controller case in Table 1 is larger than the second one of the decentralized controller. However, it is observed that with the proposed distributed controller, the damping ratios of all the oscillation modes are improved compared to the

system with decentralized controller. This effect will be investigated based on modal analysis of the system in more detail in future work. The optimization will also be further investigated by including all the eigenvalues and use different performance metric such as the damping ratio.

Table 1. Eigenvalue analysis of the test system

\begin{tabular}{|c|c|l|c|}
\hline Case & Eigenvalues & $\begin{array}{l}\text { Damping } \\
\text { Ratio }\end{array}$ & Freq. $[\mathrm{Hz}]$ \\
\hline \multirow{3}{*}{ Open Loop } & $-0.339 \pm 4.818 j$ & 0.0703 & 0.7668 \\
& $-0.405 \pm 3.265 j$ & 0.1230 & 0.5196 \\
& $-0.354 \pm 2.615 j$ & 0.1344 & 0.4157 \\
\hline \multirow{3}{*}{ Decentralized Control } & $-0.352 \pm 4.813 j$ & 0.0730 & 0.7666 \\
& $-0.411 \pm 3.260 j$ & 0.1251 & 0.5188 \\
& $-0.355 \pm 2.609 j$ & 0.1347 & 0.4152 \\
\hline \multirow{3}{*}{ Distributed Control } & $-0.355 \pm 4.769 j$ & 0.0743 & 0.7590 \\
& $-0.409 \pm 2.860 j$ & 0.1414 & 0.4553 \\
& $-0.363 \pm 2.377 j$ & 0.1509 & 0.3783 \\
\hline
\end{tabular}

Furthermore, the damping performance of the decentralized controller and the proposed distributed controller in terms of the rotor angle deviations $\Delta \delta_{i}$ and rotor speed deviations $\Delta \omega_{i}$ of each generator is also compared when a sudden load change of the load 3 is simulated as a small disturbance to the power system starting at $t=0.5$ and lasting for $1 s$ as shown in Fig. 5 - 9. In consistence with the eigenvalue analysis in Table 1 , it is observed that all the rotor angle deviations $\Delta \delta_{i}$ are damped much faster by the distributed controller compared to the one with the decentralized controller. Moreover, the damping of the rotor speed deviations are also increased for some of the generators. It can also be observed that the oscillation of the generator $G_{3}$ and $G_{4}$ have larger amplitude than the other three generators due to the assumption in the simulation that the fault occurs at the load 3 which is physically located closer to the generator $G_{3}$ and $G_{4}$.
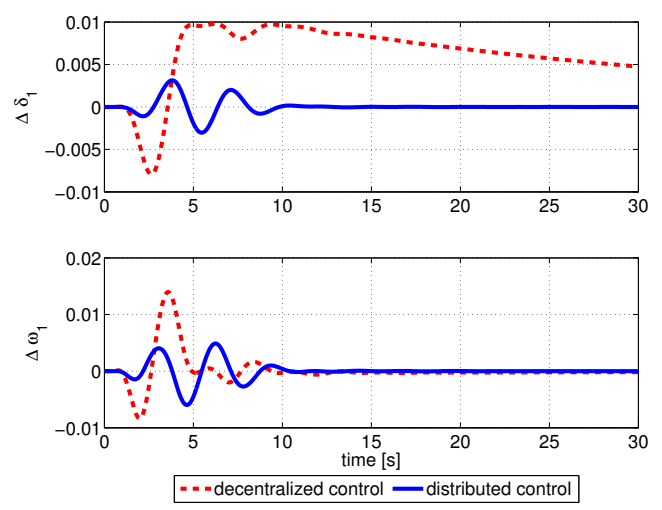

Fig. 5. Response to disturbance of the generator $G_{1}$

The integrated squared control error of the rotor angle deviations $\Delta \delta_{i}$ is shown in Table 2. It is obvious that the distributed controller achieves smaller integrated errors than the decentralized ones except for the $\Delta \delta_{4}$ of the generator $G_{4}$. This might be due to the fact that only the largest eigenvalue is considered in the optimization problem that leads to the performance degradation of a subset of the generators. A similar effect is also observed for the squared integrated control errors of the rotor speed deviations $\Delta \omega_{i}$ which is not shown here due to the space limit. 

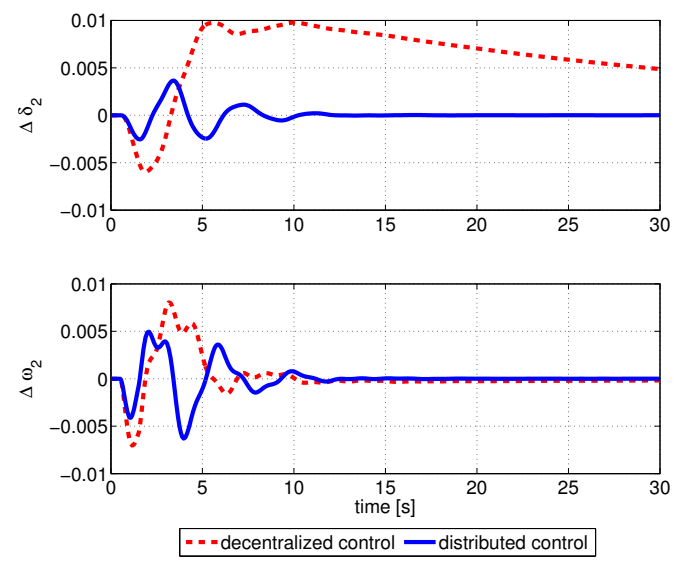

Fig. 6. Response to disturbance of the generator $G_{2}$
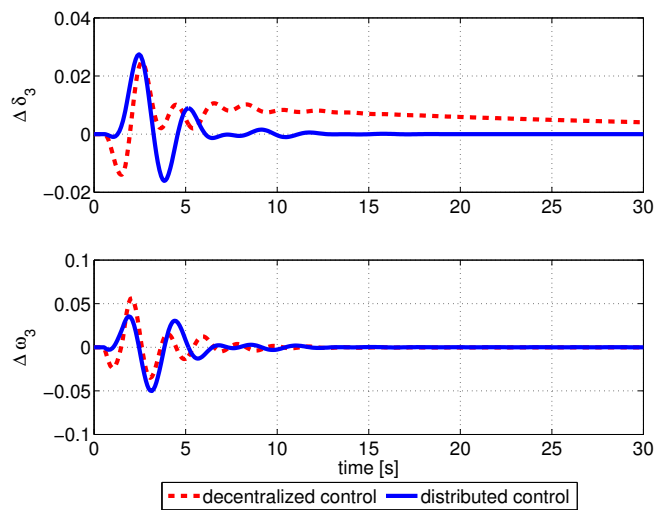

Fig. 7. Response to disturbance of the generator $G_{3}$
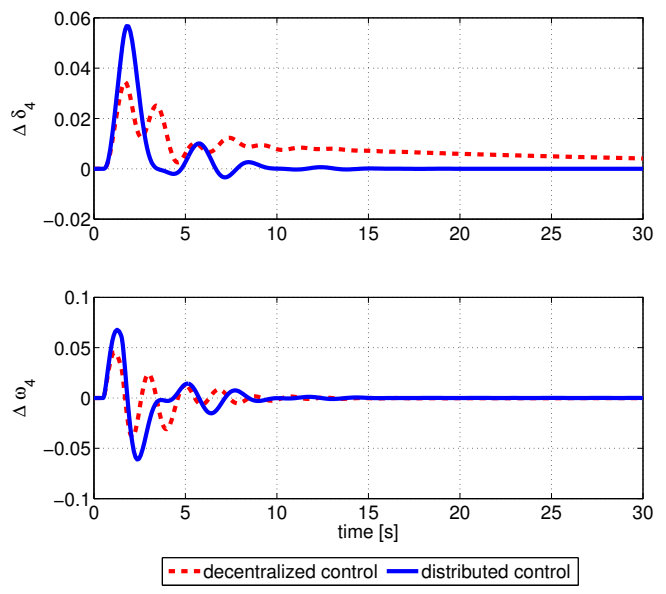

Fig. 8. Response to disturbance of the generator $G_{4}$

\section{CONCLUSION AND FUTURE WORKS}

In this paper, we proposed a design procedure for the widearea power system damping control from the aspect of a novel joint distributed optimal control and communication topology design. This approach explores the additional design degrees of freedom offered by the communication network. The problem is formulated as a mixed integer optimization problem in order to find the optimal communication topology for the distributed controller such that the decay rate is optimized under a given communication constraint. The proposed controller is evaluated in a five
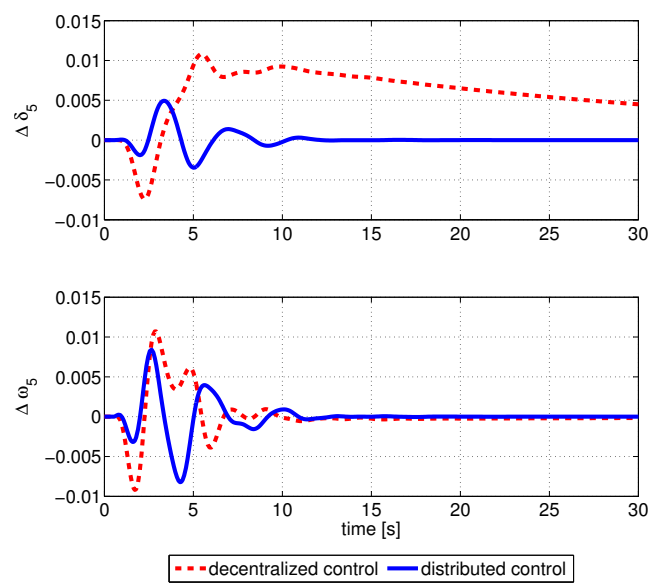

Fig. 9. Response to disturbance of the generator $G_{5}$

Table 2. Squared integrated control error of the rotor angle deviations

\begin{tabular}{|c|c|c|c|c|c|}
\hline Integrated error & $\Delta \delta_{1}$ & $\Delta \delta_{2}$ & $\Delta \delta_{3}$ & $\Delta \delta_{4}$ & $\Delta \delta_{5}$ \\
\hline Dec. Control & 0.0200 & 0.0174 & 0.0173 & 0.0357 & 0.0155 \\
\hline Dist. Control & 0.0089 & 0.0003 & 0.0002 & 0.0441 & 0.0004 \\
\hline
\end{tabular}

machine power system via a numerical simulation and it provides sufficient damping performance as shown in the results. It might open up a new perspective of control design as a combined optimization of the control and communication infrastructure for wide-area control of future power systems.

In the future, a more sophisticated objective function, i.e. another performance metric for the optimization problem in order to improve the damping performance, will be investigated. For example, instead of minimizing the eigenvalue with the largest real part of the closed loop system which may not always provide the best damping performance regarding to the other eigenvalues, in the future the optimization problem will be modified to include all the (complex) eigenvalues of the system. Moreover, the performance of the proposed technique will also be evaluated for the case of a larger power system which has more sparse structure with the inclusion of PSS.

\section{REFERENCES}

Aboul-Ela, M., Sallam, A., McCalley, J., and Fouad, A. (1996). Damping controller design for power system oscillations using global signals. IEEE Transactions on Power Systems, 11(2), 767-773.

Aldeen, M. and Crusca, F. (2002). Multimachine power system stabiliser design based on new lqr approach. IEE Proceedings in Generation, Transmission and Distribution, 494502.

De La Ree, J., Centeno, V., Thorp, J., and Phadke, A. (2010). Synchronized phasor measurement applications in power systems. IEEE Transactions on Smart Grid, 1, 20-27.

Dotta, D., e Silva, A., and Decker, I. (2009). Wide-area measurements-based two-level control design considering signal transmission delay. IEEE Transactions on Power Systems, 24(1), 208-216.

Gusrialdi, A. and Hirche, S. (2010). Performance-oriented communication topology design for large-scale intercon- 
nected systems. IEEE Conference on Decision and Control, 5707-5713.

Hashmani, A. and Erlich, I. (2008). Power system stabilizer by using supplementary remote signals. 16th Power Systems Computation Conference, July 2008 Glasgow, Scotland.

Huang, B. (2006). Stability of Distribution Systems with a Large Penetration of Distributed Generation. Ph.D. thesis, Universität of Dortmund.

Kamwa, I., Grondin, R., and Hbert, Y. (2001). Wide-area measurement based stabilizing control of large power systemsa decentralized/hierarchical approach. IEEE Transactions on Power Systems, 16(1), 136-153.

Korte, B. and Vygen, J. (2000). Combinatorial Optimization: Theory and Algorithms. Springer-Verlag.

Liu, J., Gusrialdi, A., Obradovic, D., and Hirche, S. (2009). Study on the effect of time delay on the performance of distributed power grids with networked cooperative control. 1st IFAC Workshop on Estimation and Control of Networked Systems, 168 - 173.

Lofberg, J. (2004). Yalmip: A toolbox for modelling and optimization in matlab. Proceedings of the CACSD, 284 $-289$.

Machowski, J., Bialek, J., and Dr Bumby, J. (2008). Power System Dynamics : Stability and Control. Wiley.

Ni, H., Heydt, G., and Mili, L. (2002). Power system stability agents using robust wide area control. IEEE Transactions on Power Systems, 17(4), 1123-1131.

Phadke, A. and Thorp, J. (2008). Synchronized Phasor Measurements and Their Applications. Springer.

Rostamkolai, N., Phadke, A., Long, W., and Thorp, J. (1988). An adaptive optimal control strategy for dynamic stability enhancement of ac/dc power systems. IEEE Transactions on Power Systems, 3(3), 1139-1145.

Snyder, A., Ivanescu, D., HadjSaid, N., Georges, D., and Margotin, T. (2000). Delayed-input wide-area stability control with synchronized phasor measurements and linear matrix inequalities. IEEE Power Engineering Society Summer Meeting, 2, 1009 - 1014.

$\mathrm{Wu}$, B. and Malik, O.P. (2006). Multivariable adaptive control of synchronous machines in a multimachine power system. IEEE Transactions on Power Systems, $21(4), 1772-1781$

Wu, H., Tsakalis, K., and Heydt, G. (2004). Evaluation of time delay effects to wide-area power system stabilizer design. IEEE Transactions on Power Systems, 19(4), 1935-1941.

Zhang, Y. and Bose, A. (2008). Design of wide-area damping controllers for interarea oscillations. IEEE Transactions on Power Systems, 23(3), 1136-1143.

\section{Appendix A. NOMENCLATURE}

$\Delta[\bullet] \quad$ increments of the variables

$\delta_{i} \quad$ rotor angle of $i$-th machine [rad]

$\omega_{i} \quad$ rotor speed of $i$-th machine [rad/s]

$E_{q i}^{\prime} \quad$ internal transient voltage in q-axis of $i$ th machine [p.u.]

$M_{i} \quad$ inertia constant of $i$-th machine [s]

$D_{i} \quad$ damping power coefficient of $i$-th machine [p.u.]

$X_{d i} \quad$ synchronous reactances in d-axis of $i$-th machine [p.u.]
$X_{d i}^{\prime}$

$T_{d o i}^{\prime}$

$P_{e i}$

$E_{f i}$

$b_{1 i}, b_{0 i}, c_{1 i}, c_{0 i}$

$G_{i j}, B_{i j}$

Appendix B. STATE SPACE MATRICES

$\mathbf{A}_{i}=\left[\begin{array}{ccccc}0 & 1 & 0 & 0 & 0 \\ -\frac{1}{M_{i}} \frac{\partial P_{e i}}{\partial \delta_{i}} & -\frac{D_{i}}{M_{i}} & -\frac{1}{M_{i}} \frac{\partial P_{e i}}{\partial E_{q i}^{\prime}} & 0 & 0 \\ \alpha_{i} \frac{\partial I_{d i}}{\partial \delta_{i}} & 0 & -\frac{1}{T_{d o i}^{\prime}}+\alpha_{i} \frac{\partial I_{d i}}{\partial E_{q i}^{\prime}} & \frac{b_{1 i}}{T_{d o i}^{\prime}} & \frac{b_{0 i}}{T_{d o i}^{\prime}} \\ 0 & 0 & 0 & -c_{1 i} & -c_{0 i} \\ 0 & 0 & 0 & 1 & 0\end{array}\right]$

$\mathbf{B}_{i}=\left[\begin{array}{lllll}0 & 0 & 0 & 1 & 0\end{array}\right]^{T}$,

$\mathbf{A}_{i j}=\left[\begin{array}{ccccc}0 & 0 & 0 & 0 & 0 \\ -\frac{1}{M_{i}} \frac{\partial P_{e i}}{\partial \delta_{j}} & 0 & -\frac{1}{M_{i}} \frac{\partial P_{e i}}{\partial E_{q j}^{\prime}} & 0 & 0 \\ \alpha_{i} \frac{\partial I_{d i}}{\partial \delta_{j}} & 0 & -\frac{1}{T_{d o i}^{\prime}}+\alpha_{i} \frac{\partial I_{d i}}{\partial E_{q j}^{\prime}} & \frac{b_{1 i}}{T_{d o i}^{\prime}} & \frac{b_{0 i}}{T_{d o i}^{\prime}} \\ 0 & 0 & 0 & 0 & 0 \\ 0 & 0 & 0 & 0 & 0\end{array}\right]$

with $\alpha_{i}=\frac{X_{d i}-X_{d i}^{\prime}}{T_{d o i}^{\prime}}$.

Appendix C. SYSTEM PARAMETERS

Table C.1. Generator parameters

\begin{tabular}{c|ccccc} 
& G1 & G2 & G3 & G4 & G5 \\
\hline \hline$M_{i}$ & 4.6 & 4.75 & 4.53 & 4.04 & 5 \\
$D_{i}$ & 3.14 & 3.77 & 3.45 & 4.08 & 3.5 \\
$x_{d i}$ & 0.1026 & 0.1026 & 1.0260 & 0.1026 & 1.0260 \\
$x_{d i}^{\prime}$ & 0.0339 & 0.0339 & 0.3390 & 0.0339 & 0.3390 \\
$T_{d o i}^{\prime}$ & 5.67 & 5.67 & 5.67 & 5.67 & 5.67 \\
$b_{1 i}^{\prime}$ & $6.66 K_{A}$ & $6.66 K_{A}$ & $6.66 K_{A}$ & $6.66 K_{A}$ & $6.66 K_{A}$ \\
$b_{0 i}$ & $3.33 K_{A}$ & $3.33 K_{A}$ & $3.33 K_{A}$ & $3.33 K_{A}$ & $3.33 K_{A}$ \\
$c_{1 i}$ & 33.3 & 33.3 & 33.3 & 33.3 & 33.3 \\
$c_{0 i}$ & 3.33 & 3.33 & 3.33 & 3.33 & 3.33 \\
$K_{A}$ & 200 & 200 & 200 & 200 & 200
\end{tabular}

Table C.2. Generator operating points

\begin{tabular}{c|ccccc} 
& G1 & G2 & G3 & G4 & G5 \\
\hline \hline$V$ & 1.05 & 1.03 & 1.025 & 1.05 & 1.025 \\
$\theta$ & 0 & 0.1051 & 0.0943 & 0.0361 & 0.0907
\end{tabular}

Table C.3. Parameters of transmission lines [p.u.]

\begin{tabular}{cc|ccc} 
node & node & $\mathrm{R}$ & $\mathrm{X}$ & $\mathrm{B} / 2$ \\
\hline \hline 1 & 7 & 0.00435 & 0.01067 & 0.01536 \\
2 & 6 & 0.00213 & 0.00468 & 0.00404 \\
3 & 6 & 0.02004 & 0.06244 & 0.06408 \\
4 & 8 & 0.00524 & 0.01184 & 0.01756 \\
5 & 6 & 0.00711 & 0.02331 & 0.02732 \\
6 & 7 & 0.04032 & 0.12785 & 0.15858 \\
7 & 8 & 0.01724 & 0.04153 & 0.06014
\end{tabular}

